\title{
A systematic study of X-ray variability in the ROSAT all-sky survey ${ }^{\star}$
}

\author{
B. Fuhrmeister and J. H. M. M. Schmitt
}

\author{
Hamburger Sternwarte, University of Hamburg, Gojenbergsweg 112, 21029 Hamburg, Germany
}

Received 11 December 2002 / Accepted 21 February 2003

\begin{abstract}
We present a systematic search for variability among the ROSAT All-Sky Survey (RASS) X-ray sources. We generated lightcurves for about $30000 \mathrm{X}$-ray point sources detected sufficiently high above background. For our variability study different search algorithms were developed in order to recognize flares, periods and trends, respectively. The variable X-ray sources were optically identified with counterparts in the SIMBAD, the USNO-A2.0 and NED data bases, but a significant part of the X-ray sources remains without cataloged optical counterparts. Out of the 1207 sources classified as variable $767(63.5 \%)$ were identified with stars, $118(9.8 \%)$ are of extragalactic origin, $10(0.8 \%)$ are identified with other sources and $312(25.8 \%)$ could not uniquely be identified with entries in optical catalogs. We give a statistical analysis of the variable X-ray population and present some outstanding examples of X-ray variability detected in the ROSAT all-sky survey. Most prominent among these sources are white dwarfs, apparently single, yet nevertheless showing periodic variability. Many flares from hitherto unrecognised flare stars have been detected as well as long term variability in the BL Lac 1E1757.7+7034.
\end{abstract}

Key words. surveys - X-rays: general - stars: activity - stars: flares

\section{Introduction}

The ROSAT X-ray observatory, launched in 1990, carried out an all-sky survey during its first six months of operations. This first X-ray imaging all-sky survey tremendously increased the number of X-ray sources known at the time. Variability is known to be one of the key properties of the X-ray sky. Almost all source classes, with the exception of supernova remnants, clusters of galaxies and possibly white dwarfs, show variable X-ray emission. Some source classes have already been searched for variability in the RASS data. For example, Haisch \& Schmitt (1994) studied the variability of RS CVn systems and other active giants detected in the RASS data, Greiner et al. (1999) searched for X-ray counterparts of $\gamma$-ray bursts, and individual outstanding events have been reported. Donley et al. (2002) searched systematically for galaxies with count rate variability of more than a factor of 20 by cross-correlating the RASS sources with data from pointed observations and found five galaxies in this process. Grupe et al. (2001) used a similar approach to search for variability in soft X-ray detected active galactic nuclei. Furthermore Stelzer \& Neuhäuser (2000) investigated the X-ray properties of stars in the Tucanae association using both RASS and pointed ROSAT data.

Send offprint requests to: $\mathrm{B}$. Fuhrmeister,

e-mail: bfuhrmeister@hs .uni-hamburg.de

* The complete version of Table 7 is only available in electronic form at the CDS via anonymous ftp to

cdsarc.u-strasbg.fr (130.79.128.5) or via

http://cdsweb.u-strasbg.fr/cgi-bin/qcat?J/A+A/403/247
They generated lightcurves and found flares as well as irregular variability among young stars. There are more examples for variability studies of individual sources using the RASS data (Schmitt 1994), however, up to now no systematic search for variability in the total all-sky survey data has been carried out.

The first great advantage of the RASS data set is that it is unbiased in the sense that no specific objects or regions of the sky were preferentially observed. Therefore all types of objects can be analysed for variability and a statistical analysis of the variability properties can be performed in an unbiased fashion. A second reason why the RASS data is very suitable for a variability search programs is the temporal sampling of the survey data. Due to the survey geometry all sources were scanned repeatedly for at least two days for up to about $30 \mathrm{~s}$ during each satellite orbit. Therefore the lapse time for each X-ray source is at least two days and much longer for sources close to the poles of the ecliptic. Such a temporal sampling is almost never achieved in pointed observations. Therefore for the study of variability on longer time scales there is a definite advantage of the survey data compared to pointed observations, which are of course much deeper but typically sample variability on shorter time scales.

In this paper we will elaborate on the problem of finding variability in the RASS data and identifying the variable $\mathrm{X}$-ray sources with optical counterparts. Section 2 of our paper describes the RASS data and the survey geometry, Sect. 3 presents the generation of the lightcurves and some problems arising in that process; the different search algorithms used in 
our study are explained as well. Section 4 discusses the identification of the X-ray sources with optical counterparts via the SIMBAD and USNO-A2.0 catalogs and the NED data base and provides a statistical analysis of the variable sources. Section 5 shows some examples of variability highlights we found in this survey.

\section{The survey data}

The ROSAT satellite was operated in an almost circular low Earth orbit at an altitude of $580 \mathrm{~km}$ with an inclination of $53^{\circ}$. It carried the Wide Field Camera for observations in the XUV and the X-ray telescope (XRT) for the measurement of soft X-rays in the energy range of $0.1-2 \mathrm{keV}$, corresponding to wavelengths of 120-6 $\AA$. During the scanning phase of the satellite the position sensitive proportional counter (PSPC) was mounted in the focal plane of the XRT. This is a photon counting detector which registered the arrival time of each X-ray photon, the position and the photon energy with quite modest spectral resolution.

The RASS observations were carried out between 1990 July 30 and 1991 January 25; a few gaps in the survey were filled in July 1990, in February and in August 1991. During the RASS the satellite scanned the sky in great circles. Scanning and orbital period were the same with each orbit lasting $96 \mathrm{~min}$. The scans circles were perpendicular to the plane of the ecliptic and contained the ecliptical poles. The ecliptical longitude of the instantaneous scan longitude moved approximately with the apparent speed of the Sun along the ecliptic; thus the whole sky was covered within half a year. Since the circular field of view had a radius of 57 arcmin (and the angular velocity of the Sun is $\sim 1$ degree per day) a source near the ecliptic plane was scanned for about two days while sources at the ecliptical poles were scanned during the whole survey. The period $T$ during which a given source is observed is a function of the ecliptical latitude $\beta$ and is computed as

$T \sim \frac{2}{\cos (\beta)}$.

The length of a single scan can last up to $30.4 \mathrm{~s}$ if the source passes exactly through the center of the field of view; note that the scan speed was $3.75 \mathrm{arcmin} / \mathrm{s}$. The exact length of a scan depends on the impact parameter $b$, which is defined as the nearest distance of a given source to the left (western) border of the detector during a given scan; the scan geometry is illustrated in Fig. 1.

Clearly, the RASS data for each source consist of a series of snapshots, each of which has a different exposure time (depending on its impact parameter). The individual snapshots follow each other with the orbital period of $\approx 96 \mathrm{~min}$. For a detailed description of the survey geometry and its implications on the timing analysis see Belloni et al. (1994).

\section{Data analysis}

\subsection{Source detection}

A meaningful search for variability is possible only for sufficiently strong sources. For example, a source with $0.1 \mathrm{cts} / \mathrm{s}$

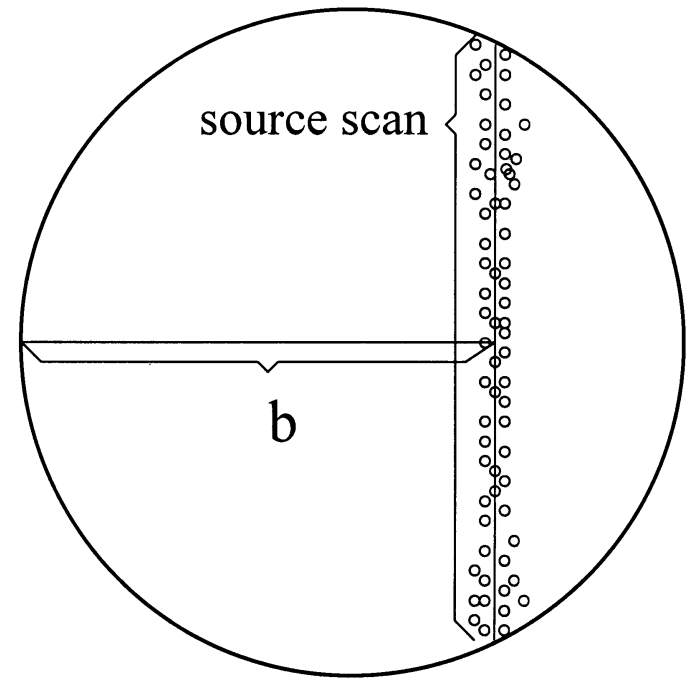

Fig. 1. Schematic view of a single scan of a source in the PSPC detector and its impact parameter $b$. The big circle indicates the detector field of view, while the little circles represent the individual photons detected from the given source. The vertical line is the calculated apparent path of the source through the detector. The actually recorded photons scatter around this regression line due to the detector's point response function.

will produce only three expected counts in the longest possible scan with an signal-to-noise ratio $(S N R)$ of less than two. In order to find sufficiently strong sources we proceeded as follows: A source detection was first carried out on the merged survey data with a maximum likelihood algorithm; an $\mathrm{X}$-ray source was accepted for further analysis if it exceeds the threshold of the maximum likelihood value of 15 (which corresponds roughly to 5 sigma). This resulted in about 30000 point sources for which lightcurves were generated. For the source detection and generation of these lightcurves the EXSAS context within MIDAS (distributed by the European Southern Observatory) was used; for description of the EXSAS environment see Zimmermann et al. (1994).

\subsection{Extraction of lightcurves}

For the construction of X-ray lightcurves we extracted all photons within a circular region centered on the nominal source position. The correct choice of the size of the extraction region is a nontrivial problem. Clearly, the larger the extraction region the more background is picked up; on the other hand, because of the sensitivity of the point response function with off axis angle, too small an extraction region leads to an unacceptable loss of source photons. Ideally one should work with a "breathing" detect cell that changes its size according to the actual off axis angle, a process computationally a bit cumbersome. Since meaningful results are expected to emerge only from stronger sources, we decided instead to work with two different extraction radii depending on the scan impact parameter $b$, one for the central scans with middle values of $b$ and one for the noncentral scans with larger and smaller values of $b$. The detect cell sizes were determined in such a way that $90 \%$ of the source 


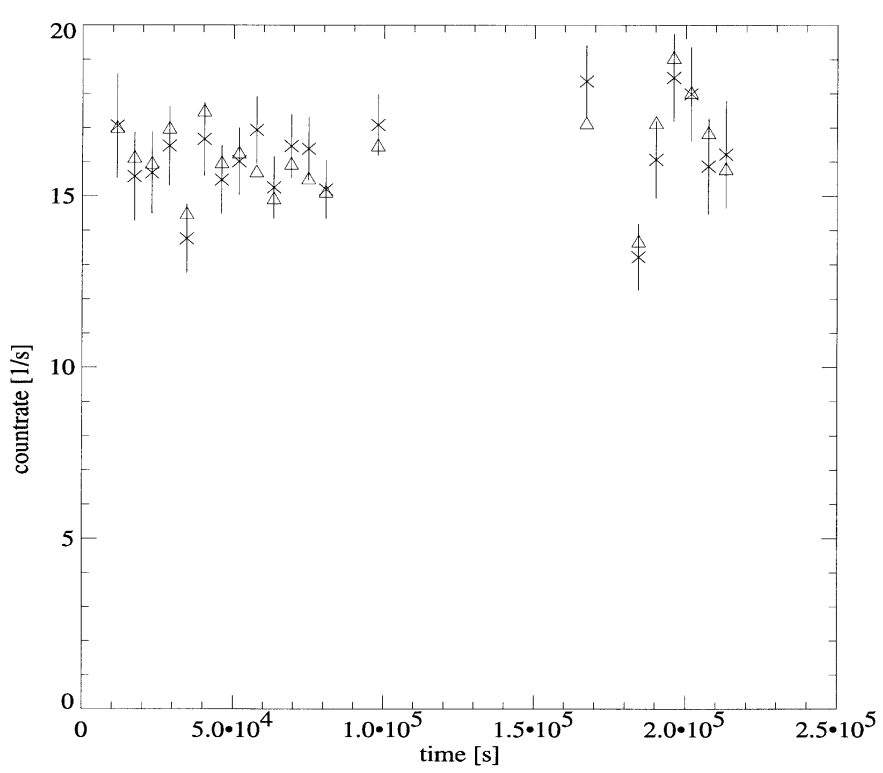

Fig. 2. Comparison between the two extraction methods applied to the strong white dwarf source Sirius B. The crosses with error bars indicate the count rates computed using a fixed radius detect cell large enough to collect more than 99 percent of the source photons. The triangles indicate the values from our adopted two radii method with a correction to account for the full photon flux.

photons must be collected; the resulting lightcurves were then corrected for the missing source photons.

We tested this method using four strong sources, i.e. Capella, Algol and the white dwarfs Sirius B and HZ 43; especially the latter two sources are expected to be constant. All of these sources are so strong that one can choose a fixed radius which collects on average more than 99 percent of the source photons while the background level is negligible. A comparison of the lightcurves generated with the two methods resulted in differences at a level of about 5 percent (see Fig. 2). Since the variability effects that can be meaningfully searched for in the RASS data are much larger we conclude that our method is acceptable and does not introduce any artificial variability. Also note that scan to scan variations of up to $20 \%$ can occur for presumably constant sources; any variability below that level may therefore be spurious.

For the generation of the lightcurves we used the EXSAS procedure create/accepted, which computes the exposure time per scan from the known scan speed and the average photon impact parameter $b$ of the scan. Since for many scans there are only a few photons defining the scan path on the detector plane, this method is not very exact. To improve the accuracy of the impact parameter the procedure automatically performs a linear fit of $b$ against the average photon arrival time $T$ of each scan. The relation between $b$ and $T$ is theoretically an arc of a sinusoid, but a straight line approximation is good enough for all sources with a distance of more than 2 degrees from the ecliptic poles. In the immediate vicinity of the ecliptic poles the approximation of a straight line breaks down, because the sources are detected in every scan. Hence the center position of the source in each scan is the projection of the circular motion of the source onto the detector and therefore a sinusoid.

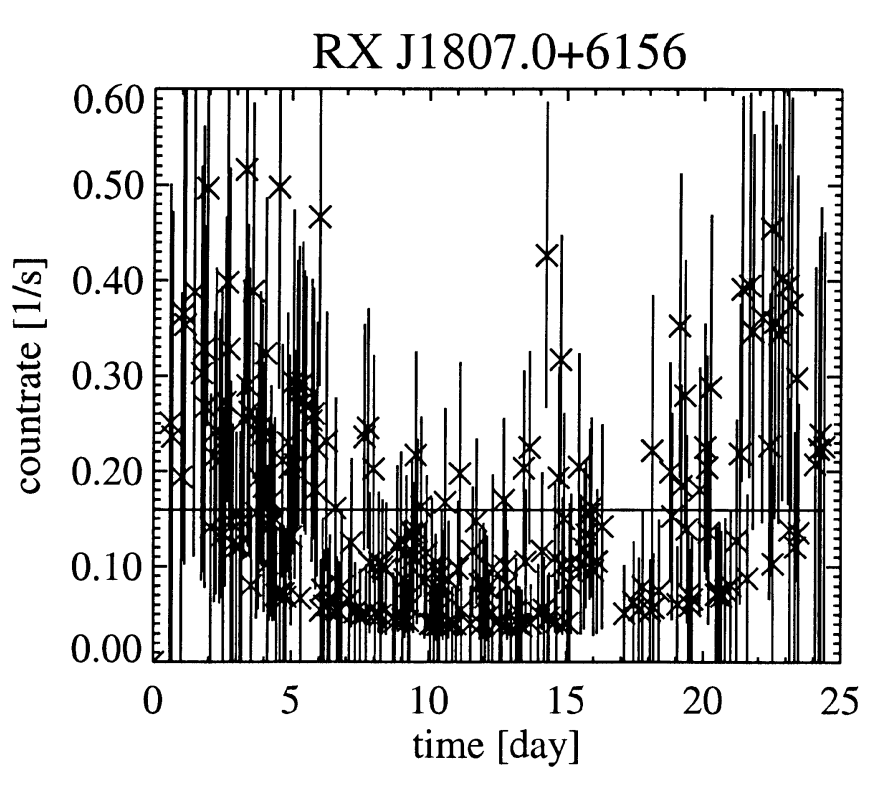

Fig. 3. Example of a RASS lightcurve showing a typical curvature with the count rate rising towards both start and end of the observations; note that scans with zero counts and zero exposures are not included.

A second reason led us to excluded these sources from our analysis. All these sources turned out to be very weak and for such sources at a distance of less than about 15 degrees of the ecliptical poles there is another problem illustrated in Fig. 3.

Sources with an average count rate of less than $\sim 0.5$ photons per second show a typical curvature that increases with decreasing count rate. This curvature is not caused by an incorrect vignetting correction, rather it is due to the survey geometry and the small number counting statistics: The exposure time in the scans near the edge of the detector and therefore at beginning and end of the survey observations is very short. If a single photon or maybe two are detected in one of these scans, the count rate is consequently high. If, on the other hand, no photon is detected, no impact parameter and no data points can be derived with our method. Near the ecliptic poles the impact parameter $b$ changes only slowly from scan to scan thus causing the described curvature of the lightcurves. In principle, the same effect is also present for sources at lower ecliptic latitudes but it does not become apparent because of the rapid change of impact parameter $b$ with increasing scan number. In order to confirm this interpretation we simulated lightcurves where the photons for each scan were computed assuming a fixed count rate under Poisson noise. For these artificial sources lightcurves were generated showing the same curvature as observed in the ROSAT lightcurves, too. The curvature could be removed by averaging over several scans at the expense of temporal resolution. However, since we have no easy way to distinguish between scans with zero counts and scans with zero exposure we decided not to pursue this matter any further. Unfortunately the lightcurves with curvature turned out to be a major problem for our variability search algorithms, since both spurious flares and periods were recognised by our search algorithms. 


\subsection{Search for flares}

For the search for flares we developed an algorithm combining different tests. Altogether ten tests were applied, and if five give a positive answer then the lightcurve is assumed to be variable. Out of the ten tests seven are empirical and three are statistical ones. The statistical ones are the Kolmogorov-Smirnov test and the $\chi^{2}$-test with a double weight. For a description of these two tests see e.g. Press et al. (1992). For the KolmogorovSmirnov test a false-alarm probability threshhold of 40 percent was used. This high threshhold can be used because the KStest is only one indicator for a flare out of ten. Using a lower threshhold makes the KS-test insensitive to minor flares. The $\chi^{2}$-test was adapted using

$\chi^{2}=\frac{1}{n} \cdot \sum_{i=1}^{n} \frac{\left(\mu-r_{i}\right)^{2}}{e_{i}^{2}}$

with $\mu$ being the mean, $r_{i}$ being the count rate in the $i$ th scan and $\mathrm{e}_{i}$ being the error of the count rate. If $\chi^{2}>1.8$ then the scan with the largest deviation is searched and excluded. A new $\chi^{2}$ is computed and the process is iterated until $\chi^{2}<1.5$. If one of the excluded scans has a count rate higher than the mean, then the test is positive. If the excluded scans have all count rates lower than the mean the lightcurve is not thought to be variable since dips can be instrumental. The two threshholds for $\chi^{2}$ were empirically determined with a testfield. The empirical tests try to identify signatures in the lightcurves which have the typical shape of a flare. Specifically, we searched for a time bin with a count rate three times higher than the median, for four time bins following each other, each higher than the median and each one lower than the prior one.

We tested our search algorithm with simulated lightcurves. First we created constant lightcurves with only Poisson noise imposed and tested the number of lightcurves associated with spurious variability. We realized - not surprisingly - that this kind of error depends on the mean count rate. With a high count rate (10 counts per second) about 4 percent of the simulated lightcurves are classified as variable, while with a low count rate $(<0.3$ counts per second) about 0.3 percent is classified as variable despite their constancy. The bent lightcurves we found (see Sect. 3.6) were not taken into account in this test.

\subsection{Search for periods}

The search for periods was performed with the Lomb-Scargle Algorithm (see Press et al. 1992), that can deal with discrete, unevenly spaced data. The algorithm computes a periodogram, in which the amplitude translates directly into a false-alarm probability of the apparent period. The threshold for the falsealarm probability for variable sources was set to 5 percent. Moreover the found period must not be longer than 95 percent of the lightcurve to be accepted.

\subsection{Search for trends}

In lightcurves with ecliptic latitude $\beta \gtrsim|60|^{\circ}$ corresponding to a minimum of four days observation was also searched for
Table 1. Number of variable sources found in the RASS. In brackets the number of sources researched by eye. In the lower part are the numbers of sources which show two types of variability.

\begin{tabular}{|c|c|c|c|}
\hline \multicolumn{3}{|c|}{ number of sources analysed } & 29970 \\
\hline & $\beta \gtrsim|60|^{\circ}$ & $\beta \lesssim|60|^{\circ}$ & total \\
\hline flares & $330(664)$ & 664 & 994 \\
\hline periods & $85(887)$ & 60 & 145 \\
\hline trends & $90(412)$ & - & 90 \\
\hline flares and trends & 2 & & \\
\hline periods and trends & 2 & & \\
\hline flares and periods & 18 & & \\
\hline
\end{tabular}

trends. The algorithm compared the median of the first quarter of the lightcurve with the median of the last quarter of the lightcurve. If the difference exceeds 30 percent of the median of the whole lightcurve and is at least 0.2 then a trend is assumed.

The sources with ecliptical latitude $\beta \lesssim|60|^{\circ}$ were not searched for trends since the lightcurves consist of only few scans so that the the median of the lightcurve becomes less robust.

\subsection{Revision by eye}

The analysis of the lightcurves was performed in two steps. First, all lightcurves with ecliptical latitude $\beta \gtrsim|60|^{\circ}$ were analysed, then the much shorter ones at lower latitudes. We found it necessary to visually inspect all high latitude lightcurves because of the curvature feature described above. The ill defined background level could produce spurious flares and the curvature in the high latitude lightcurves would produce spurious periods. Therefore spurious variability arising from such curved lightcurves was removed manually. The data quality of lightcurves at low ecliptical latitude turned out to be much better and we did not find it necessary inspect all the lightcurves by eye. The number of variable sources found at both low and high ecliptic latitudes is given in Table 1 . Table 1 shows very clearly that at low ecliptic latitudes flares are detected almost exclusively; at high latitude this pattern changes completely, however, we also found it necessary to reject a significant number of sources that probably show only spurious variability.

\section{Optical identification of the variable sources}

In order to perform a statistical analysis of the variable sources it is necessary to identify them with optical counterparts. For this purpose we used three catalogs, i.e. the SIMBAD database, the USNO-A2.0 catalog and the NED. SIMBAD (Set of Identifications, Measurements, and Bibliography for Astronomical Data) contains names, positions and known properties of 3063000 objects outside the solar system that are mainly galactic. The USNO-A2.0 catalog of the U.S. Naval Observatory contains 526280881 stars with positions and brightness in the $R$ and $B$ band. The NED (NASA/IPAC Extragalactic Database) contains names, positions and basic data for about 4700000 extragalactic objects. 
Table 2. At the left are shown the limits of the X-ray to $V$ band flux ratio found in the identified sample. In the middle the same for the sample Krautter et al. (1999) used. At the right the used threshold for the $R$ band.

\begin{tabular}{lcc|cc|c}
\hline \hline Object class & \multicolumn{2}{c}{$\log \left(\frac{f_{X}}{f_{V}}\right)$} & \multicolumn{2}{c}{$\log \left(\frac{f_{X}}{f_{V}}\right)$} & \multicolumn{2}{c}{ used } \\
& $\min$ & $\max$ & $\min$ & $\max$ & threshold \\
\hline B stars & -3.09 & -0.56 & - & - & -0.56 \\
A stars & -3.58 & -3.41 & -4.59 & -3.78 & -3.41 \\
F stars & -4.19 & -1.92 & -4.82 & -1.97 & -1.92 \\
G stars & -4.37 & -1.84 & -5.52 & -0.80 & -0.80 \\
K stars & -3.52 & -0.10 & -4.57 & +0.20 & +0.20 \\
M stars & -1.99 & +1.30 & -4.41 & -0.14 & -0.14 \\
\hline
\end{tabular}

\subsection{Identification with the SIMBAD database}

Since we expect mostly stars to show variability (flares and periods) we first tried to identify the detected variable sources with counterparts cataloged in the SIMBAD database. In order to match RASS sources with SIMBAD entries we used a search radius of 1.5 arcmin which significantly exceeds the error circle of $90 \%$ of 30 " determined by Krautter et al. (1999). Despite this rather generous search radius many X-ray sources turned out to have no cataloged SIMBAD counterpart.

\subsection{Identification with the USNO-A2.0 catalog}

For the sources that remained unidentified with SIMBAD entries or that were only listed as X-ray sources in SIMBAD we tried to find counterparts using the USNO-A2.0 catalog. In order to limit the number of counterparts to a reasonable level we constrained the search radius to $40^{\prime \prime}$, a procedure that left us with at least one candidate object for each source. In order to assess the plausibility of this matching procedure by positional coincidence we estimated the ratio of the X-ray flux to the $R$ band flux and defined a threshold above which the candidate stars were not accepted. The X-ray flux was estimated from mean count rate using the conversion factor $\alpha=6 \times 10^{-12} \frac{\mathrm{erg}}{\text { counts } \mathrm{cm}^{2}}$. The flux $f_{\lambda}(R)$ in the $R$ band outside the atmosphere was computed from $\log f_{\lambda}(R)=-0.4 m_{R}-8.58+3$ where $m_{R}$ is the brightness in the $R$ band; the width of the $R$ band was assumed to be $1000 \AA$. The value 8.58 applies only for B stars and changes slightly with the spectral class; the exact numbers can be found in Allen (1973). Note that for $\mathrm{O}$ stars the equation is not valid; however, since variability among O-type stars is quite unusual this should hardly matter.

In order to define sensitive thresholds we used typical maximal values of this ratio known in the $V$ band from Krautter et al. (1999) and of our own sample that was identified with SIMBAD. In our own sample we find a tendency to higher values for both the lower and the upper limits of the ratio which we ascribe to flares in this sample that increment the X-ray flux. The determined values of the ratio in the $V$ band as well as the used threshold in the $R$ band are listed in Table 2 .

Using these threshold values out of 436 X-ray sources not cataloged as SIMBAD entry $183 \mathrm{X}$-ray sources ended up with exactly one acceptable object within the search radius while 149 objects had no acceptable entry within the search radius.

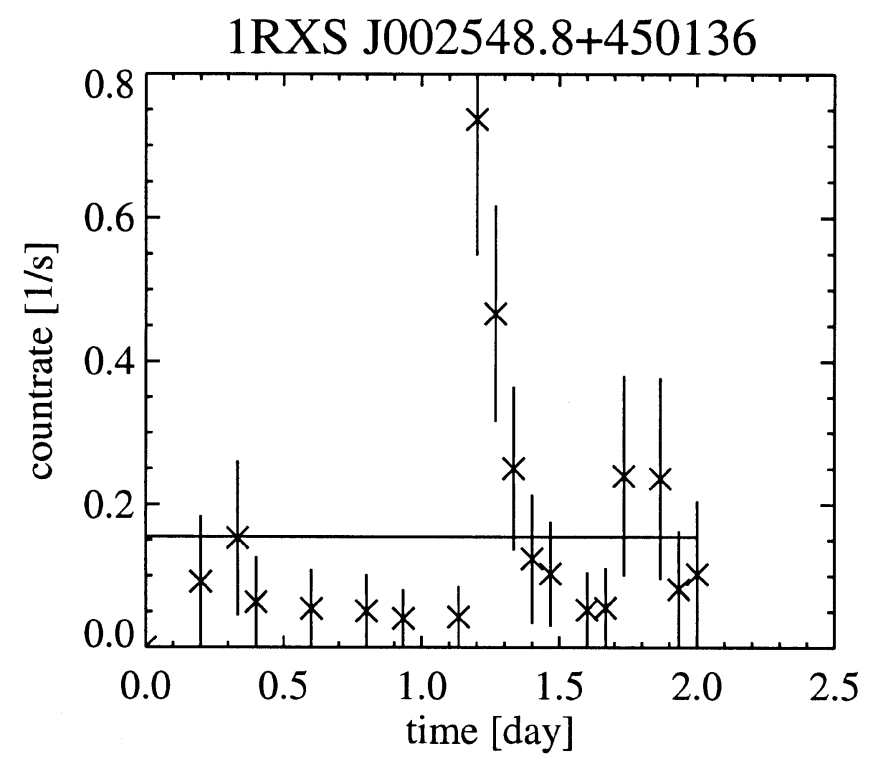

Fig. 4. Example of a typical lightcurve of an optical not cataloged source. Note that strong flare with its sudden rising and long lasting decay strongly suggesting a stellar nature of this event.

In all other cases more than one acceptable star was found in the search radius so no unique (positional) identification can be performed.

\subsection{Uncataloged objects}

Since the number of objects with no stellar counterpart in the USNO catalog is quite high these objects must either be very active in the X-ray band or very faint in the $R$ band or they are no stars at all. In order test this hypothesis we used the NED data base. For the 149 objects without stellar identification we could identify 15 with galaxies and 4 with quasars listed in the NED data base but not in SIMBAD; the remaining 130 sources are still unidentified. We suggest that these are likely to be stars for the most part due to two reasons. First there are stellar counterparts for all sources found in the USNO-A2.0 catalog without applying the threshold ratio of the X-ray to visual flux. Taking into account that most of the unidentified objects show flares the threshold is presumably too low and therefore the stellar content of the sample is underestimated. The second reason is implied by the lightcurves themselves: Many of the unidentified sources do in fact show typical stellar flare lightcurves as shown in Fig. 4; it would be a conspiracy of nature if the counterparts were not stars.

In Table 3 we give a summary of the identifications obtained from the SIMBAD, USNO-A2.0 and NED data bases. The variable objects are distinguished according to their stellar types as well as to the type of variability found; note that several types of variability can be found for the same lightcurve. The quoted percentages refer to the percentage of the respective source class among all the variable sources. As is obvious from Table 3, late-type stars are responsible for the largest portion of the variable source population; about $10 \%$ of the variable sources are of extragalactic origin. 
Table 3. The identification of the variable sources. Given are the numbers of objects of each object type for the different kinds of variability. In brackets is shown the percentage. AGN is active galactic nuclei, LXB is low mass X-ray binary, SNR is supernova remnant, WD is white dwarf. The sources in the category "not identified with cataloged objects" are mainly identified with X-ray sources, with some minor identification of infrared and radio sources. The objects in the category "stars" are stars from the SIMBAD database with unknown spectral type and stars from the USNO catalog where more than one star with a sensible X-ray to optical flux ratio was found. The sources not uniquely identified with SIMBAD are found in the category "not uniquely identified".

\begin{tabular}{ccccc}
\hline \hline Object class & total & flares & periods & trends \\
\hline Sy1 & $60(5.0)$ & 44 & 6 & 12 \\
Sy2 & $3(0.2)$ & 0 & 1 & 2 \\
AGN & $36(3.0)$ & 24 & 5 & 7 \\
Gal & $19(1.6)$ & 10 & 3 & 6 \\
& & & & \\
LXB & $8(0.7)$ & 7 & 0 & 1 \\
SNR & $2(0.2)$ & 1 & 0 & 1 \\
& & & & \\
WD & $13(1.1)$ & 9 & 5 & 1 \\
O & $2(0.2)$ & 2 & 0 & 0 \\
B & $9(0.7)$ & 8 & 2 & 1 \\
A & $12(1.0)$ & 10 & 2 & 0 \\
F & $45(3.7)$ & 40 & 3 & 2 \\
G & $95(7.9)$ & 77 & 13 & 7 \\
K & $239(19.8)$ & 205 & 27 & 10 \\
M & $137(11.3)$ & 134 & 2 & 1 \\
star & $215(17.8)$ & 191 & 22 & 9 \\
& & & & \\
not uniquely & & & & \\
identified & $173(14.3)$ & 144 & 22 & 8 \\
not id with & & & & \\
cataloged obj & $139(11.5)$ & 88 & 32 & 22 \\
total & 1207 & 994 & 145 & 90 \\
\hline
\end{tabular}

A list of all ROSAT all-sky survey variable sources is given in Table 7, available at the CDS, where we list the name, position, type and the luminosity (if known) of the objects. Furthermore there are variability flags given showing which sort of variability was found. The flags indicate the following: fa - flare automatically found, f - flare, pa - period automatically found, $\mathrm{p}$ - period, ta - trend automatically found, $\mathrm{t}$ - trend.

\subsection{Unexpected identifications}

X-ray variability among late-type stars does not come unexpectedly. However, there are some variable objects found in classes that are not expected to show any variability at all. The former are the classes SNR and WD, the latter are the classes of stars with spectral type O, B and possibly A. A closer inspection of the individual sources shows the two O-stars to be high mass X-ray binaries (HMXB) as well as 4 of the B-stars (and one of the WDs); the specific sources are V779 Cen, 4U 2206+54(O-stars), V662 Cas, LMC X-3, GP Vel, 2A 0116-737 (B-stars) and Her X-1 (WD). Further, two of the B-stars are double or multiple stars, namely CCDM J20016+7027AB and HD 5394, while 5 of the A-stars are
Table 4. Remarks on the objects for some peculiar classes.

\begin{tabular}{ccc}
\hline \hline Object class & Number & Remarks \\
\hline O-stars & 2 & 2 HMXBs \\
B-stars & 9 & 4 HMXBs \\
& & 2 variable X-ray sources \\
& & 2 double star \\
& & 1 SNR (high count rate) \\
A-stars & 12 & eclipsing binaries \\
& & 4 Novastar \\
& & 2 known X-ray sources \\
SNR & 2 & 2 high count rate \\
WD & 13 & 1 high count rate \\
& & 4 periods (discussed later) \\
& & 2 binary \\
& &
\end{tabular}

eclipsing binaries of Algol type (HD 18022, HD 139319, HD 153345, V752 Sqr, HZ Dra). Some of the A-stars are identified using only the USNO-A2.0 catalog and the threshold for the X-ray to optical flux ratio; the spectral type is therefore quite uncertain. The other early-type stellar sources are X-ray sources listed in the SIMBAD database (HD 21364 and HD 33904 are B-type stars, HD 43940 and HD 116160 are A-type stars, the nova-like star is the CV BL Hyi)

All of the variable SNRs (SNR 021.0+63.0, SNR 051969.0 and SNR 0525-66.0) and some of the WDs have high count rates and therefore a relatively wide dispersion in the data which can trick especially the flare search algorithm (cf. Fig. 2). The four WDs for which periods have been found will be discussed below. One WD has an M-type companion (IN CMa), two others (WD 0809-728 and WD 1648+407) show small and rather long term variability that cannot be explained by high count rates or known physical properties. Out of these two WD $1648+407$ is suspected to be a binary (Green et al. 2000), but this needs confirmation. We checked for stellar counterparts of these objects in the USNO-A2.0 catalog. A search with a 1.5 arcmin radius revealed in both cases nearby red stars, so these two white dwarfs might be misidentifications. Remarks on these objects can be found in Table 4 . In addition to these object classes there are three objects for which in SIMBAD only galaxy clusters or groups are found as possible counterparts. On the other hand, the X-ray lightcurves of at least two of these three objects show flares and look in fact quite stellar. It was confirmed with the USNO-A2.0 catalog that there are possible stellar counterparts for these objects. We therefore placed these three objects in the category "not uniquely identified".

\section{Some examples of variability}

In addition to the statistical analysis of the variable sources we present some highlights of variability detected in the RASS data; because of our specific interest in stars and the broad variety of variable X-ray sources this selection is subjective by necessity. We focus on objects where the detection of variability is mostly due to the long observation times of the RASS, i.e., 


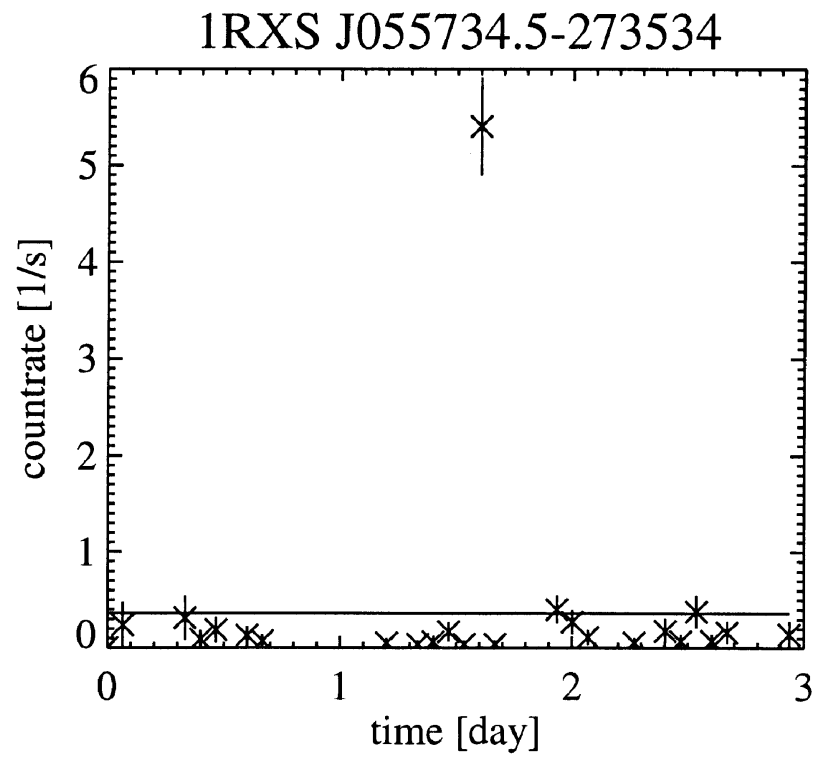

Fig. 5. The RASS lightcurve for 1RXS J055734.5-273534 with a short duration flare detected only in one single scan. Note the enormous peak X-ray flux of this event.

variability that would have probably remained undiscovered in (shorter) pointed data.

Note: The solid line in all the lightcurve plots indicate the mean count rate.

\subsection{New X-ray flare stars}

Many X-ray flares were detected on objects that could either not be identified with optical counterparts or could only be identified with stars listed in the USNO-A2.0 catalog. Both groups contain objects that show long duration flares with both the flare onset and the whole decay observed as well as objects that show sporadic short flares with very high apparent fluxes. Some of these short duration events were already found by Greiner et al. (1999) in their search for $\gamma$-ray bursts, but due to their decision of studying only objects with a count rate consistent with zero before and after the flare they in fact missed many actual flares (albeit no $\gamma$-ray bursters). These short flares are often significantly detected only in one time bin with possibly more than one hundred photons in this bin. An example for such an event on an essentially anonymous object without identification in the USNO-A2.0 catalog is shown in Fig. 5. The quiescent count rate is about $0.1-0.2$ counts/s rising to more than 5 counts/s in the scan covering the flare. From the available data it is not possible to tell whether the data point is in the flare rise, peak or decay. The two scans following the flare are somewhat elevated, so that this event could also be interpreted as along duration flare, but these count rate enhancements are not significant.

There are more such outstanding flare events detected only in one scan, but even more interesting are the newly detected long duration flares. Some of these belong to previously known flare stars. In Fig. 6 we present the RASS lightcurve of the nearby (optical) flare star GJ 3305. This star was observed twice during the RASS, the first time in the beginning of the

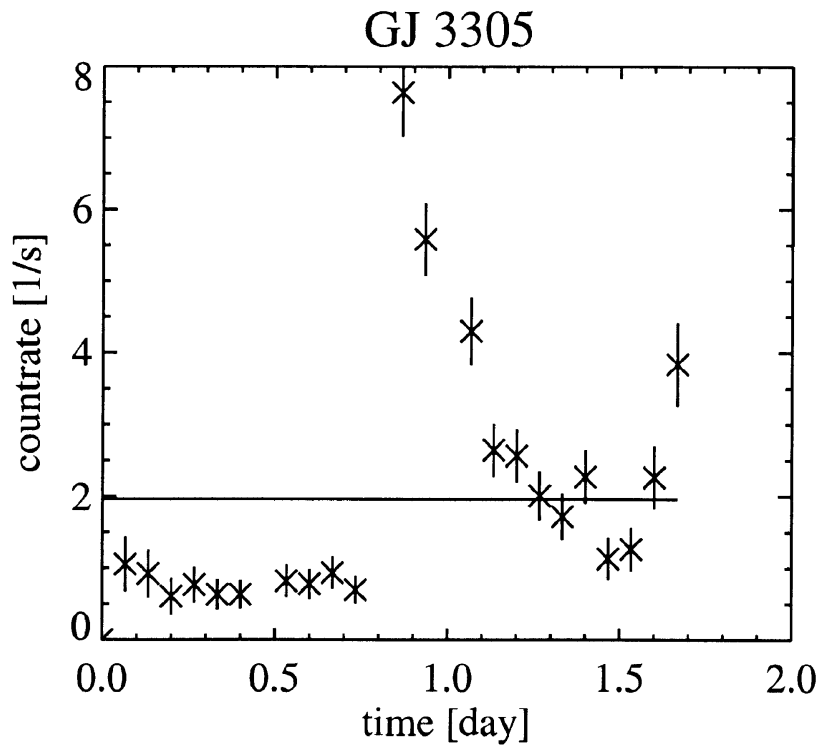

Fig. 6. The lightcurve of GJ 3305. The long duration flare is fully observed including both onset and the whole decay. Since the distance towards GJ 3305 is known, the energetics of the flare can be calculated (cf. text for details).

all-sky survey showing no significant variability, and second time at the end of the all-sky survey showing this spectacular flare. Note that the flare decay lasts about one day and produces yet another example of a long-duration flare on M-type stars.

We fitted the lightcurve of this flare with an exponential function

$c(t)=A_{0} \mathrm{e}^{-\frac{t}{\tau}}+B$

where $c(t)$ is the count rate as function of time $t, B$ the quiescent background count rate, $\tau$ the flare decay time and $A_{0}$ the count rate at flare peak. The parameters $\tau$ and $A_{0}$ determine the radiated flare energy, which is given by

$E=A_{0} \tau \alpha \cdot 4 \pi R^{2}$

where $R$ is the distance of the star and $\alpha$ the above mentioned conversion factor. The two parameter $A_{0}$ and $\tau$ were fitted while the background $B$ was fixed to the median. From the best fit parameters $A_{0}=7.4 \pm 1.1$ and $\tau=27633 \pm 8205$ and using the distance of $15.2 \mathrm{pc}$ (Jahreiss et al. 1998) we find a total released energy of $3.4 \times 10^{34} \mathrm{erg}$. This number exceeds the typically quoted soft X-ray energy releases of the largest solar flares by more than two orders of magnitude, but compares well to other flares observed on M-type stars, for example the long-duration flare on EV Lac described by Schmitt (1994).

In addition to flares from previously known flare stars we report the detection of long duration flares on previously unknown flare stars with counterparts only in the USNO-A2.0 catalog. The lightcurves of two such objects are shown in Figs. 7 and 8.

\subsection{Periodic variability}

Periodic variability was found for a substantial number of Xray sources belonging to different source classes. In addition 


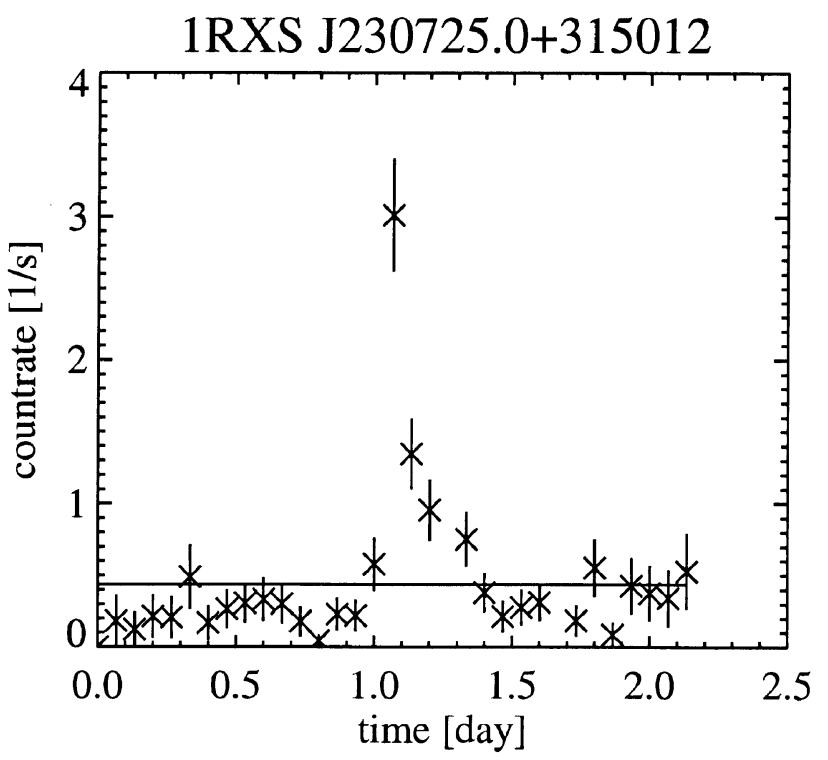

Fig. 7. The RASS lightcurve of an anonymous star. It is tentatively identified with a K star listed in the USNO-A2.0 catalog.

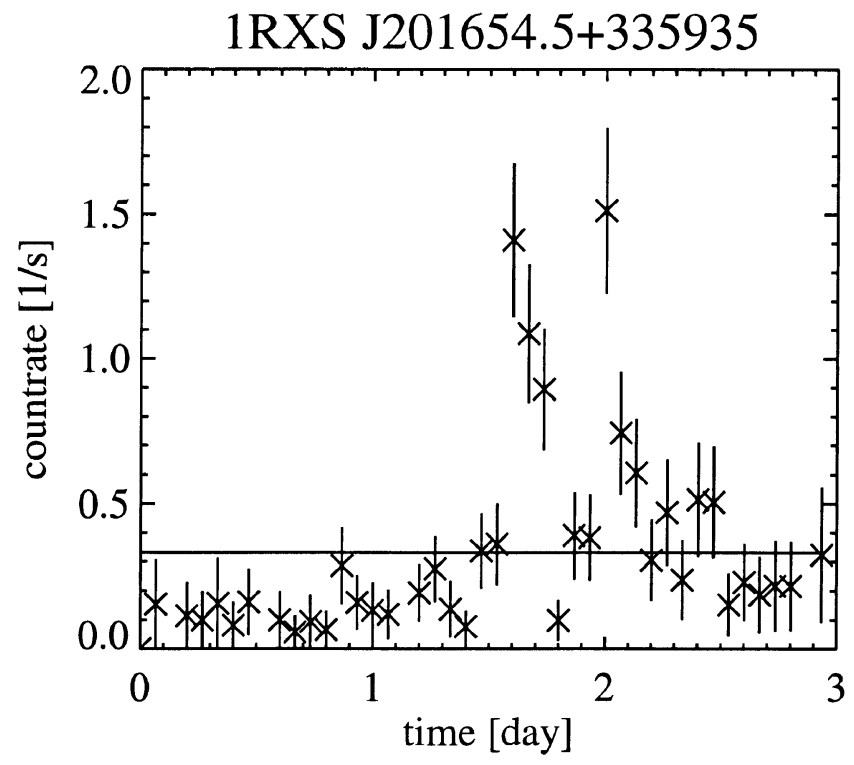

Fig. 8. The RASS lightcurve of an anonymous star. It is not identified uniquely in the USNO-A2.0 catalog. Note the multiple flare event which is quite typical for stellar sources.

to periodic variability in short-period binary systems like RS $\mathrm{CVns}$ or $\mathrm{CV}$ s, we also find periodic variability in sources where such variability is uncommmon and hard to understand. We specifically report periodic variability for the galaxy RBS 1490 and for four white dwarfs detected in our survey. While periodic variability among active galaxies is rare and unusual, quite number of the X-ray emitting WDs appear to be periodic. The four WDs in question are all previously known white dwarfs and all are very soft X-ray sources as expected for white dwarfs, therefore a misidentification is unlikely. The results of our period searches for white dwarfs are given in Table 5; the error of the period is computed from the estimated FWHM of
Table 5. Designations of the four periodically variable WDs, the RASS determined periods and the false-alarm probability (fap) in percent.

\begin{tabular}{lcc}
\hline \hline Name & Period [h] & fap \\
\hline WD 1057+719 & $25.3 \pm 6.3$ & 2.1 \\
GD 394 & $26.8 \pm 3.0$ & 1.2 \\
FBS 1500+752 & $11.6 \pm 0.3$ & 4.3 \\
1ES 1631+78.1 & $69.4 \pm 8.3$ & 4.4 \\
\hline
\end{tabular}

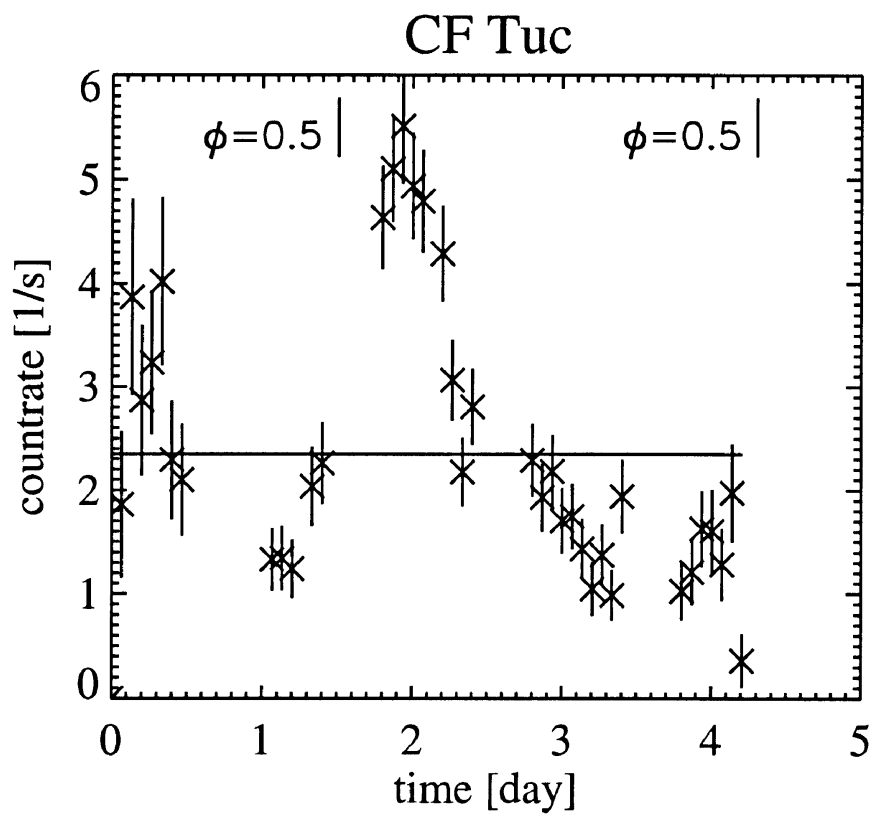

Fig. 9. RASS lightcurve of the active binary CF Tuc.

the peak in the periodogram. Below we discuss the sources individually.

\subsubsection{The active binary CF Tuc}

CF Tuc (HD 5303) is a partially eclipsing RSCVn system whose components are of spectral type G0IV and K4IV (Coates et al. 1983). For this system an orbital period of 2.798 day is known from $U B V$ photometry (Budding 1985), the rotation of the two system components is synchronised with the orbital period. The RASS X-ray lightcurve of CF Tuc is shown in Fig. 9; its high degree of variability is self-evident. Our $\mathrm{X}$ ray period of $2.7 \pm 0.8$ days is in full agreement with the optically determined period. In Fig. 9 we also marked the times of optical secondary minimum as computed from the ephemeres HJD $2444555.009+2.7977672 \mathrm{~d} \times \mathrm{E}$ (Kürster \& Schmitt 1996). Clearly, the X-ray minima do not coincide with any optical minimum. Alternatively, the RASS lightcurve of CF Tuc can also be interpreted as a long duration flare with a duration length close to the optical period; while this appears to be a strange coincidence, CF Tuc is known to be capable of producing even much longer flares (Kürster \& Schmitt 1996). 


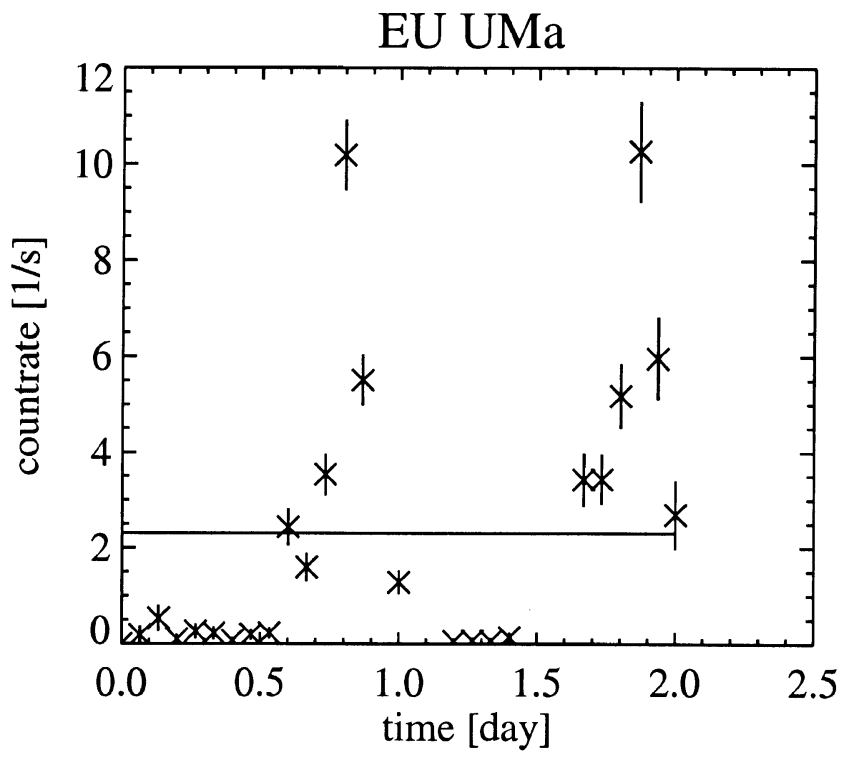

Fig. 10. RASS lightcurve of the cataclysmic variable EU UMA.

\subsubsection{The cataclysmic variable EU UMa}

The RASS lightcurve of the cataclysmic variable of AM Her type EU UMa is in Fig. 10. One clearly sees a period of roughly one day (24.9 hours), which is much larger than periods typically found for CVs and specifically the EU UMa system period of 90.144 minutes (Ritter \& Kolb 1998). Following Mittaz et al. (1992), who present the ROSAT-WFC survey data for this source, we interpret the observed period $P_{\text {obs }}$ as a result of folding the satellite period of $P_{\text {sat }}=96.2 \mathrm{~min}$ with the binary period $P_{\text {bin }}$ according to the rule $P_{\text {bin }}^{-1}=P_{\text {sat }}^{-1} \pm P_{\text {obs }}^{-1}$. Taking this aliasing into account, the ROSAT survey data result in a binary period of $90.36 \mathrm{~min}$, consistent with the WFC and optical data of EU UMa.

\subsubsection{The galaxy RBS 1490}

Another peculiar case of a RASS lightcurve is the case of the galaxy RBS 1490, which shows a period of 12.2 hours; the lightcurve is shown in Fig. 11. Since the variability amplitude is very high (more a factor of 5 variations from peak to peak) the period is extremely significant, on the other hand, such a temporal behaviour is not expected even for a Seyfert 1 galaxy. Therefore we checked for the presence of stars in the USNO-A2.0 catalog and found four stars in an area within a 1.5 arcmin search radius around the X-ray position. All of those stars have too high a ratio of their X-ray to visual fluxes and we thus reject those stars as possible counterparts. We provide the positions and provisional spectral types of those stars in Table 6 for further investigation and conclude that at present no satisfactory explanation for the RASS lightcurve of RBS 1490 can be given.

\subsubsection{The white dwarf 1 ES $1631+78.1$}

The white dwarf 1ES $1631+78.1$ is known to be a double system with the binary being a dM4 (Catalan et al. 1995).

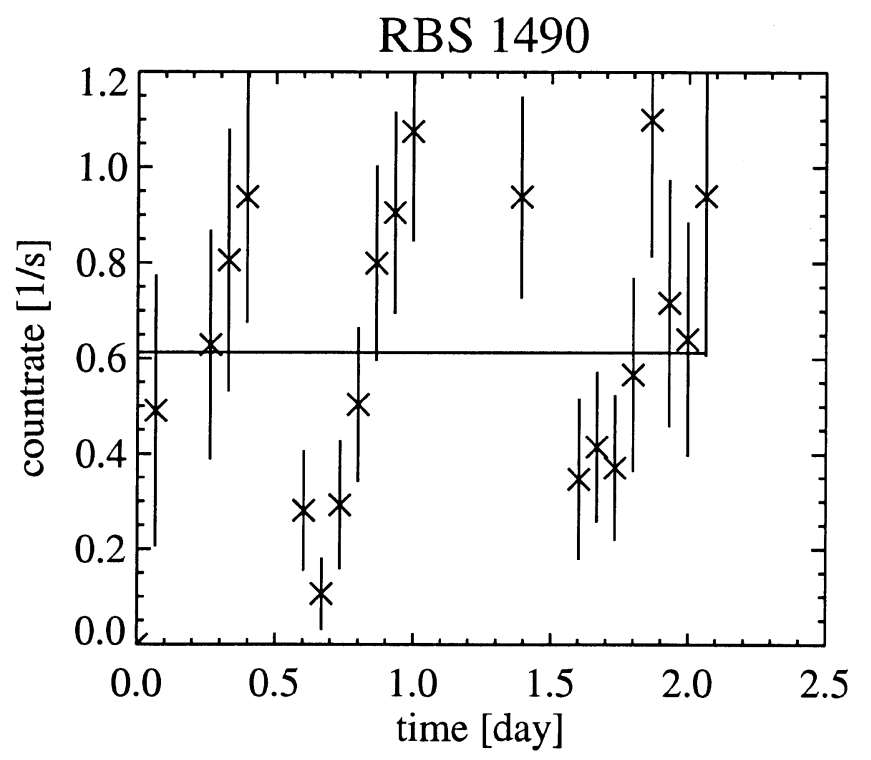

Fig. 11. RASS lightcurve of the galaxy RBS 1490.

Table 6. Positions (RA and $\delta$, epoch 2000) and optical to X-ray flux ratios for the four USNO stars in the vicinity of the source RBS1490.

\begin{tabular}{lcc}
\hline \hline Position & spectral type & $\log \left(\frac{f_{X}}{f_{V}}\right)$ \\
$152217.376+164828.39$ & $\mathrm{~A}$ & 0.39 \\
$152215.519+164825.98$ & $\mathrm{~K}$ & 0.65 \\
$152215.092+164916.63$ & $\mathrm{~F}$ & 1.72 \\
$152213.462+164901.42$ & $\mathrm{~A}$ & 0.59 \\
\hline
\end{tabular}

The period found from the RASS data is $69.4 \pm 8.3$ hours with a false-alarm probability of 4.4 percent. This white dwarf ("meaty") was used as calibration source for the Wide Field Camera (WFC) observing in the extreme ultraviolet region during the ROSAT mission. Besides a couple of short pointed exposures there is also a $36 \mathrm{ksec}$ calibration exposure of this source. Unfortunately there is a data gap in this exposure and the duration of the gap corresponds roughly to the found period. No periodic variability is found in this lightcurve, but the length of the data gap prevents us from ruling out the $69.4 \mathrm{hr}$ period found in the survey data.

\subsubsection{The white dwarf GD 394}

The lightcurve of GD 394 is shown in Fig. 13; the period of 26.8 hours is found with a false alarm probability of $1.2 \%$. The white dwarf GD 394 is also known to be variable in the EUV although it seems to be single (Dupuis et al. 2000). Analysing data from the EUVE satellite Dupuis et al. found a period of $1.150 \pm 0.003$ days, in perfect agreement with the period of $1.1 \pm 0.1$ days we found in the RASS data. Dupuis et al. interpret this period with a dark spot on the surface of the WD. They discuss two possible mechanisms that could produce such a spot: First, a magnetic field and asymmetric accretion of material along this field and second a comet may have been accreted. Since the data reported by Dupuis et al. were taken in 1995 and the RASS data were taken in 1990, we conclude that 


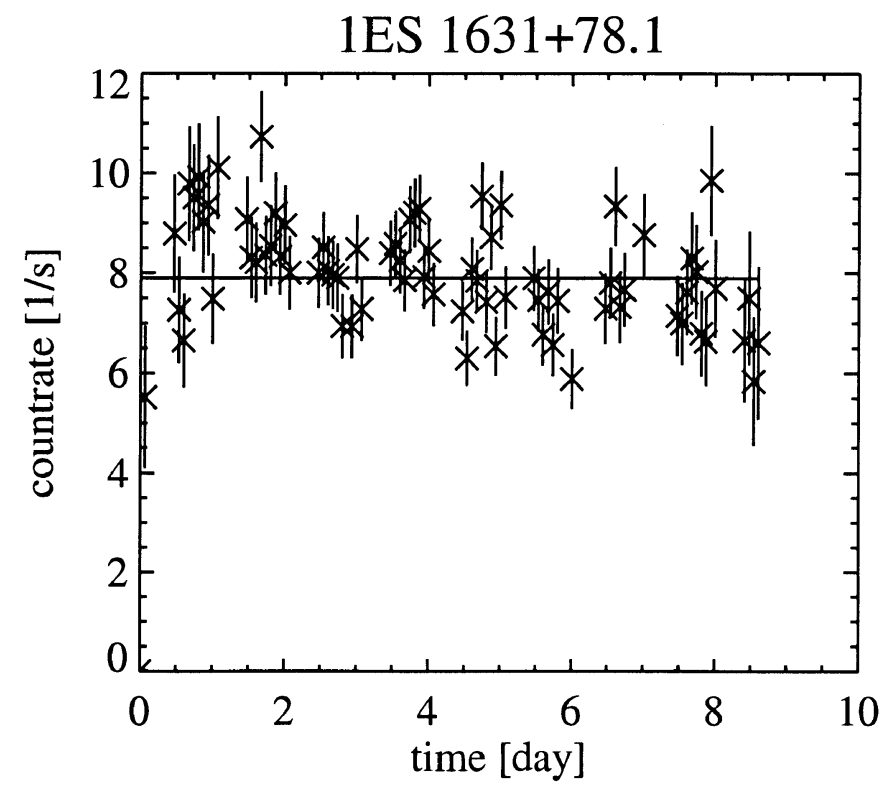

Fig. 12. RASS lightcurve of the white dwarf 1ES 1631+78.1.

GD 394

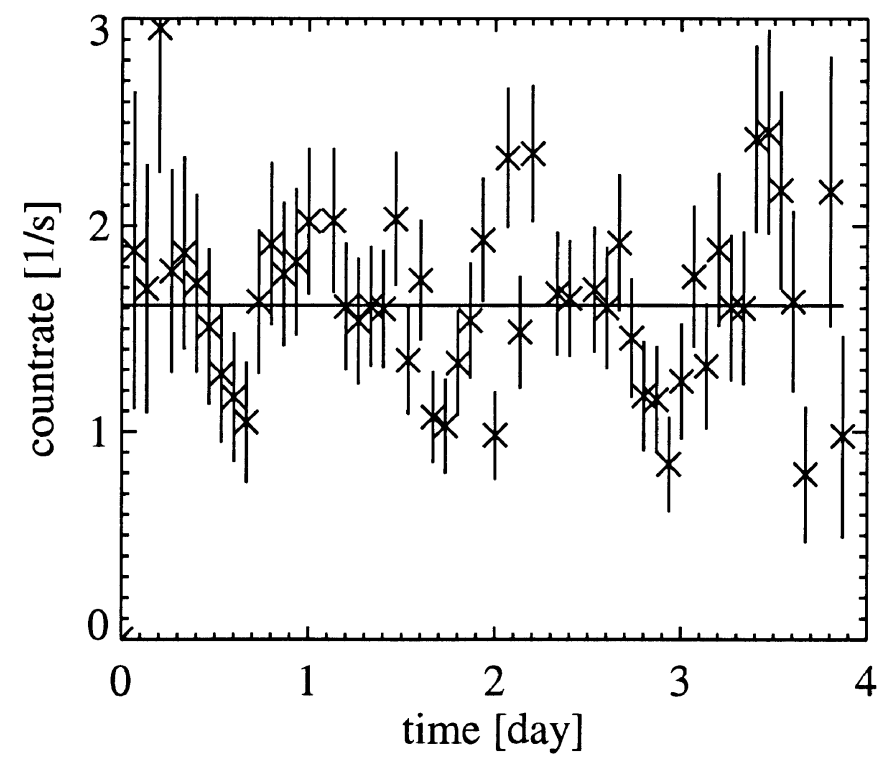

Fig. 13. RASS lightcurve of the white dwarf GD 394.

the spot has to be a persistent feature with a life time of at least 5 years which argues against interpretations invoking transient events.

\subsubsection{The white dwarf WD $1057+719$}

Another periodically variable white dwarf is WD $1057+719$; its lightcurve and period $(25.3 \pm 6.3$ hours $)$ are strongly reminiscent of GD 394; its lightcurve is shown in Fig. 14. Like GD 394 WD 1057+719 seems to be single since Green et al. (2000) and we suggest it to be an analog to GD 394.

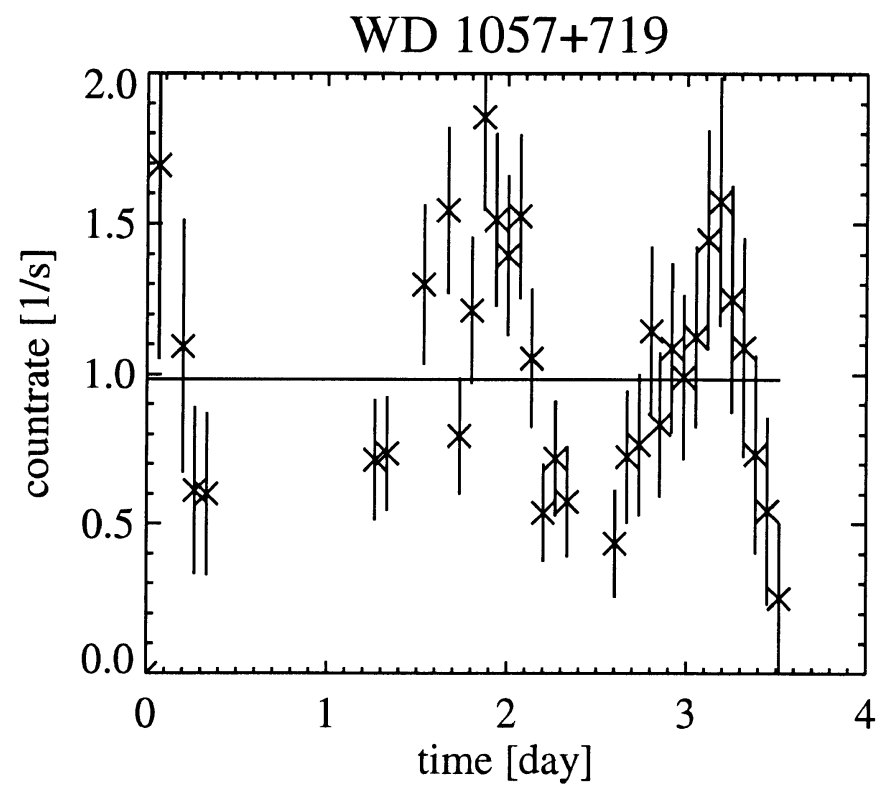

Fig. 14. RASS lightcurve of the white dwarf WD1057+719.

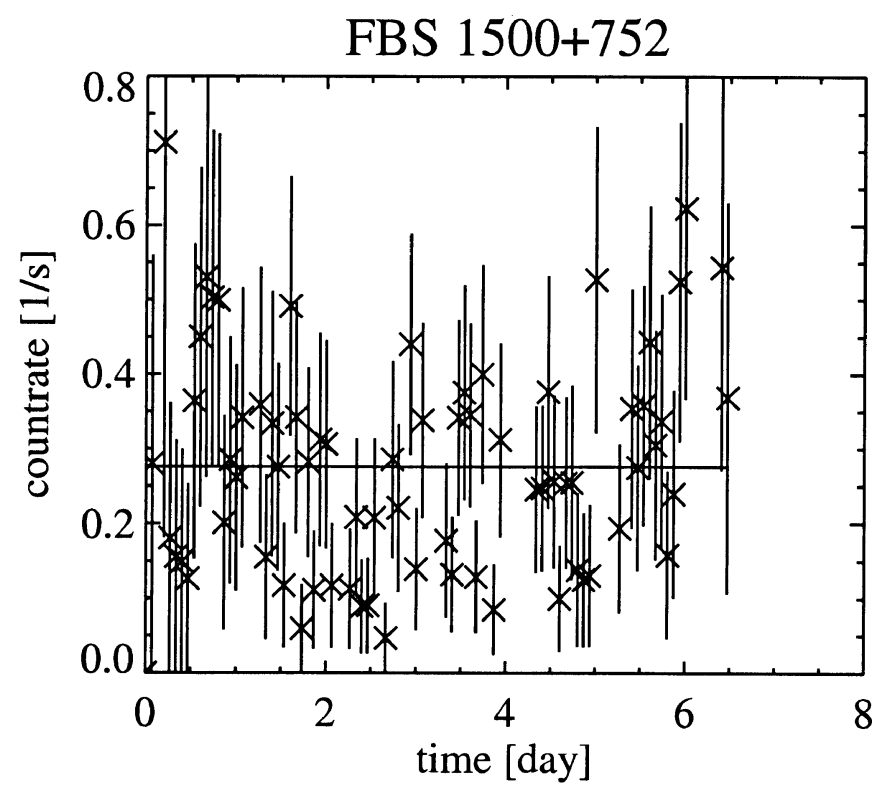

Fig. 15. RASS lightcurve of the white dwarf FBS 1500+752.

\subsubsection{The white dwarf FBS $1500+752$}

Very little is known about the object FBS $1500+752$. It is cataloged as a white dwarf in SIMBAD but no further information is available. We verified that the source is supersoft. The lightcurve of this object can be seen in Fig. 15. Our period search resulted in a period of 11.6 hours with a false alarm probability of $4 \%$. Of the four white dwarfs with periodic variability, FBS $1500+752$ is by far the weakest and the lightcurve is quite noisy, but definitely variable. Further observations are clearly needed to confirm the variability found in the RASS data. 


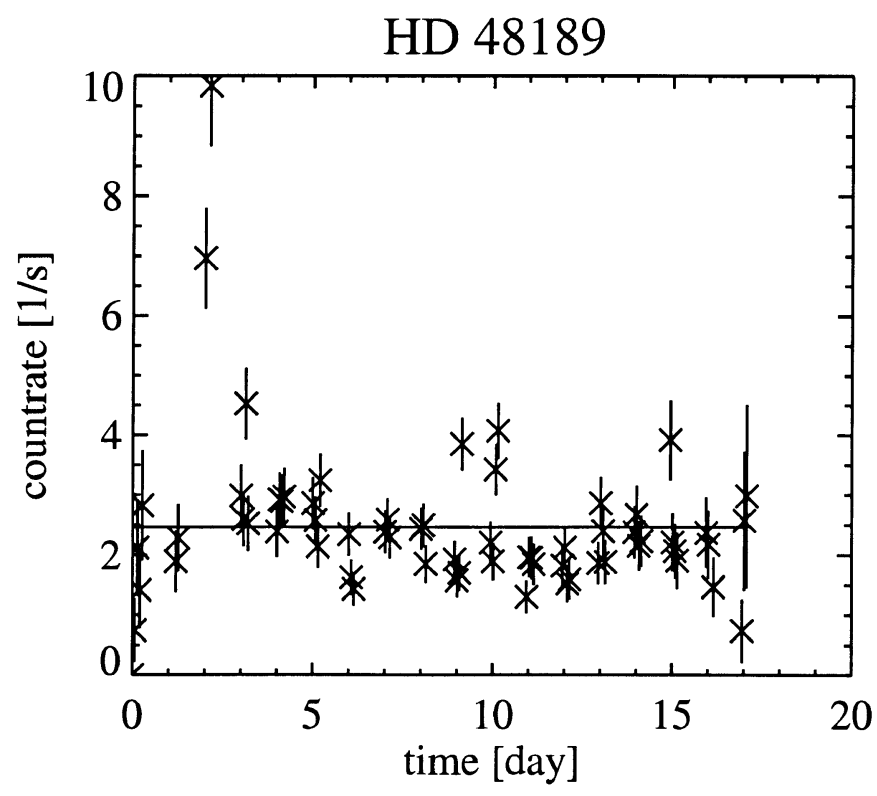

Fig. 16. Lightcurve of HD 48189. Apparently there is a flare and a period in the lightcurve.

\subsubsection{Periods not found: The case of HD 48189}

Our period search algorithm has problems finding periods in lightcurves contaminated with flares or dips. Consider the the case of HD 48189, whose lightcurve is shown in Fig. 16. HD 48189 is a relatively nearby (its HIPPARCOS parallax corresponds to a distance of $22 \mathrm{pc}$ ) visual binary with a lithium content and high X-ray luminosity $\left(L_{X}=10^{29.8} \mathrm{erg} / \mathrm{s}\right)$. Both properties indicate youth. The X-ray lightcurve of HD 48189 is characterised by two episodes of flaring which is not unexpected for alike stars. Somewhat unusually, there also is an underlying sinosoidal variation with a period of about 12.5 days in the data. This period is not found by our algorithm with the full data set, however, once the flares are manually removed, the period does show up significantly. Of course, such a procedure is - strictly speaking - not legitimate; nevertheless, it is very attractive to interpret the 12.5 day period as the rotation period of HD 48189. However, Wichmann et al. (2003) determined a $v \sin (i)$ value of $17.0 \mathrm{~km} \mathrm{~s}^{-1}$ from a high-resolution spectrum of HD 48189. Assuming a solar radius for HD 48189 we deduce a maximum period of 3 days in conflict with the measured X-ray period of 12.5 days. We thus conclude that the X-ray period does not reflect the rotation period of HD 48189. We note in passing that the spectrum obtained by Wichmann et al. (2003) (available under web site http: //www.hs. uni-hamburg.de/DE/For/ $\mathrm{Gal}$ /Xgroup/projekte.html under topic young stars) does not show any evidence for binarity of HD 48189.

\subsection{Long term trends in active galactic nuclei}

Two galaxies with long trends were detected that would not have been found in considerably shorter observations. One is the Seyfert I galaxy RBS 1660 with a lightcurve covering about 7 days (see Fig. 17); its X-ray flux first decreases for about three

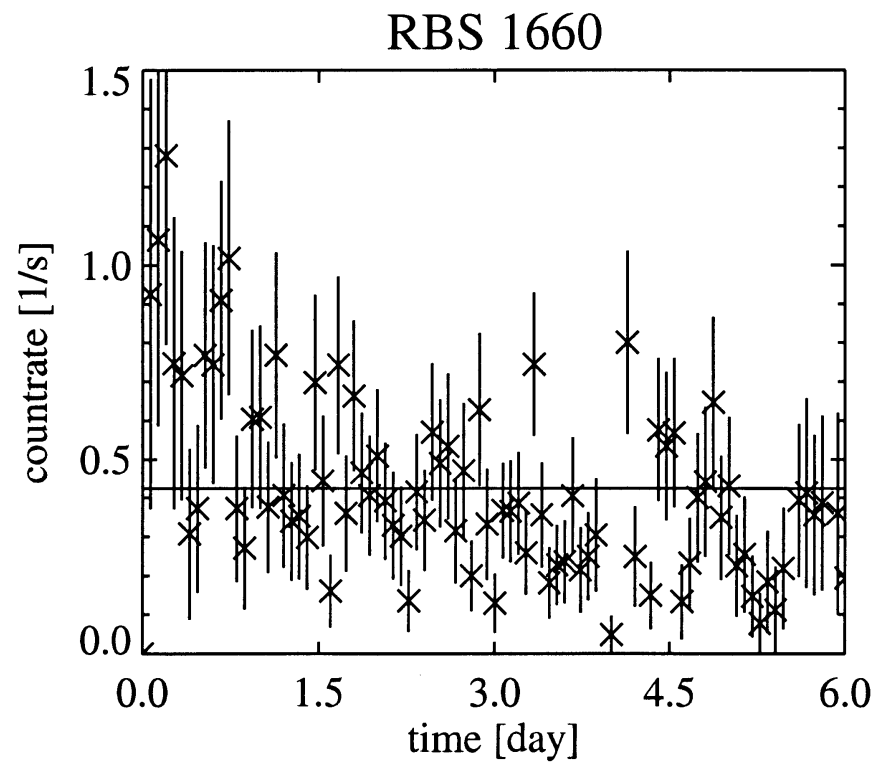

Fig. 17. The RASS lightcurve of the Seyfert 1 galaxy showing besides minor short term variability a long term weak trend.

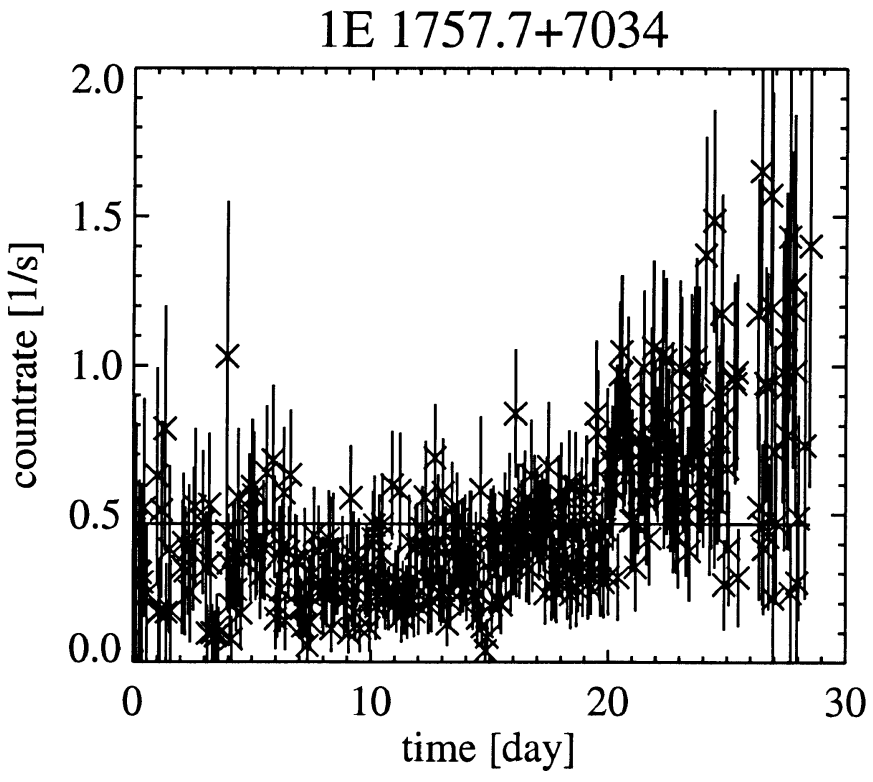

Fig. 18. The RASS lightcurve of the BL Lac showing a long term trend 1 E1757.7+7034 with an increase in count rate of a factor of $\approx 3$ over twenty days.

days, after the minimum it exhibits even shorter time-scale variability with a sharp increase by a factor of 2 . The other object is the well known BL Lac 1E1757.7+7034 with lightcurve spanning 29 days shown in Fig. 18. For 1E1757.7+7034 the $\mathrm{X}$-ray flux stayed constant for about 2 weeks, after that it start a more or less monotonic increase over the rest of the all-sky survey observations; 1E $1757.7+7034$ is known to show intraday variability in the optical (Heidt \& Wagner 1998) as well, but no X-ray variability has been reported so far. 


\section{Discussion and conclusions}

Our study revealed widespread variability among the X-ray sources detected in the RASS data. Stellar sources account for the largest portion of the variable source population. Among the stellar sources we found different types of variability: High mass X-ray binaries with a high degree of well studied variability but also flare stars, $\mathrm{T}$ Tauri stars or cataclysmic variables. Unexpected variability behavior was, however, also found: Some active galactic nuclei showed a decrease or increase in their count rate persisting throughout a couple of days. Further, we found four white dwarfs showing periodical changes in their lightcurves at high confidence levels. One of these white dwarfs is known as a double system, a second one has been very little studied so far, while the remaining two white dwarfs seems to be single; the mechanism(s) producing a periodic modulation of the X-ray flux of single white dwarfs can only be speculated upon.

Clearly the generous search radius used for our SIMBAD identifications could introduce a bias toward galactic sources since SIMBAD contains more galactic then extragalactic entries. In order to assess this possible bias we checked a random sample of stars being identified with the help of SIMBAD for extragalactic counterparts in the NED applying the same 1.5 arcmin search radius we used for SIMBAD identifications. We found that for at most 15 percent of the SIMBAD identifications an alternative NED identification could be made on positional grounds. However, since the probability for a variable X-ray source being a star is very high we conclude that the number of incorrect identifications of our variable X-ray sources must be small.

Interestingly and to us surprisingly, many of the variable $\mathrm{X}$-ray could not be identified with SIMBAD cataloged sources; these sources had therefore not been identified as "interesting" (from their variability behavior) so far. To overcome this problem we attempted to identify these X-ray sources unknown to SIMBAD with stars from the USNO-A2.0 catalog using sensible thresholds for the $\mathrm{X}$-ray to visual flux ratio. The main problem with this approach is the optimal choice of the threshold. We applied a very conservative threshold with the consequence that especially for flaring M-stars it is likely that the stellar content of the unidentified sample is underestimated.

The mean magnitude of those stars identified with USNO catalog entries is about 16 mag in the $B$-band and about 14 mag in the $R$-band. Other than their apparent luminosity very little is known about those faint stars, in particular there is no information on distances either from parallaxes or from spectroscopy; this is unfortunate because it prevents us from accurately assessing the energetics of the observed flares. The peak fluxes of some of the detected flares suggest very high energies, but distances are needed to confirm this suspicion. For some of the brighter stars HIPPARCHOS parallaxes are known. For those stars we computed the flare energy release from the fitted flare decay lightcurves and found energy releases between $10^{33}$ and $10^{35} \mathrm{erg}$. Forthcoming astrometric missions like DIVA or GAIA will provide distances for many of the stars identified with USNO entries, eventually allowing to compute the released energies.

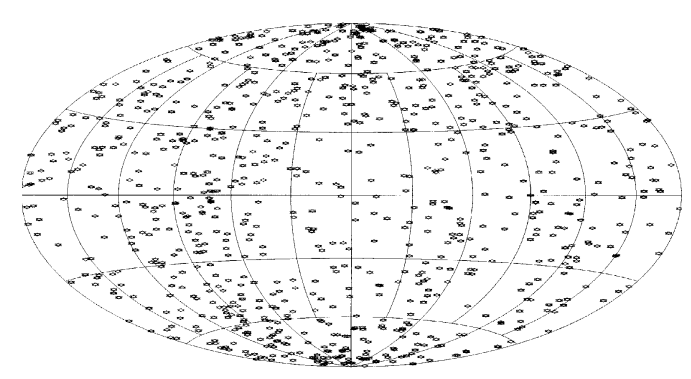

Fig. 19. Aitoff projection of the flare sources on the sky drawn in ecliptical coordinates; note the concentration towards the poles of the ecliptic which can be ascribed to the RASS survey geometry.

An Aitoff projection of the flare X-ray sources in ecliptical coordinates supports the view that most of these sources are nearby stars (cf. Fig. 19). The surface distribution is relatively uniform with an increase of sources towards the ecliptical poles. This is obviously a selection effect: Since the observation times increase for sources near the ecliptical poles, the chance for finding variability increases as well.

A natural question arising with these faint sources is their nature and age. Since these stars are quite active as evidenced by their strong flares we suspect them to be also rather young, yet no obvious clustering of the flare sources (e.g. in open clusters, star forming regions etc.) is observed (cf. Fig. 19). Apparently these stars are field stars of an young, but otherwise unspecified age. Possibly they represent the lower mass extension of the young field star population studied by Wichmann et al. (2003).

In addition to many flares on hitherto unrecognized flare stars we also find periodical variability on some stars. In a few cases these periods can be traced back to the binary periods in known binary systems. In other systems the periods are new and might be due to rotational modulation caused by active regions on the surface. The periods found range between 10 and 770 hours although we have searched for periods up to 1000 hours; the mean period is about 130 hours which is certainly consistent with the interpretation as rotational modulation. We nevertheless have to state that it is rather difficult to find rotationally modulated signals in the X-ray emission of stars except for known eclipsing systems. Since active stars, which the RASS data is most sensitive to, first, show a lot of intrinsic variability and, second, have active regions which may be predominantly concentrated near the stellar poles, rotationally modulated signals are expected to be weak and washed out by stochastic variability.

Acknowledgements. We have made extensive use of the ROSAT Data Archive of the Max-Planck-Institut für extraterrestrische Physik (MPE) at Garching, Germany. We particularly thank Rainer Gruber at MPE for technical support with EXSAS. This research has made extensive use of the SIMBAD database, operated at CDS, Strasbourg, France. This research has made use of the NASA/IPAC Extragalactic Database (NED) which is operated by the Jet Propulsion Laboratory, California Institute of Technology, under contract with the National Aeronautics and Space Administration. This research has made use of the USNO-A2.0 catalog using the VizieR search page (Ochsenbein et al. 2000). 
Table 7. Catalog of variable sources.

\begin{tabular}{|c|c|c|c|c|c|c|c|c|c|c|c|c|c|}
\hline \multirow[b]{2}{*}{ Num } & \multicolumn{2}{|c|}{$\begin{array}{ll}\text { position in X-ray } \\
\end{array}$} & \multirow[b]{2}{*}{$\mathrm{f}$} & \multirow[b]{2}{*}{ fa } & \multicolumn{3}{|c|}{$\overline{\text { flags for variability }}$} & \multirow[b]{2}{*}{ ta } & \multirow[b]{2}{*}{ type } & \multirow[b]{2}{*}{ name } & \multirow[b]{2}{*}{$\begin{array}{c}B \\
{[\mathrm{mag}]}\end{array}$} & \multirow[b]{2}{*}{$\begin{array}{c}V \\
{[\mathrm{mag}]}\end{array}$} & \multirow[b]{2}{*}{ remarks } \\
\hline & $\begin{array}{c}\alpha_{2000} \\
{[\mathrm{~h} \mathrm{~m} \mathrm{~s}]}\end{array}$ & $\begin{array}{l}\delta_{2000} \\
{\left[{ }^{\circ},{ }^{\prime \prime}{ }^{\prime \prime}\right]}\end{array}$ & & & $\mathrm{p}$ & pa & $\mathrm{t}$ & & & & & & \\
\hline 0001 & 001031.14 & +105834.5 & 1 & 1 & 0 & 0 & 0 & 0 & Sy1 & QSO B0007+107 & 0.0 & & \\
\hline 0002 & 001124.67 & -112838.5 & 1 & 1 & 0 & 0 & 0 & 0 & star & $\mathrm{V} * \mathrm{WW}$ Cet & 9.3 & 9.3 & dwarfnova \\
\hline 0003 & 001234.60 & -041630.1 & 0 & 0 & 1 & 1 & 0 & 0 & starU & 1RXS J001234.8-041633 & 0.0 & 0.0 & \\
\hline 0004 & 001309.50 & +053553.7 & 1 & 1 & 0 & 0 & 0 & 0 & $\mathrm{~K} 4 \mathrm{~V}$ & V*DV Psc & 11.79 & 10.59 & eclbinary \\
\hline 0005 & 001355.33 & -744123.8 & 1 & 1 & 0 & 0 & 0 & 0 & $\mathrm{G} 6 \mathrm{~V}$ & HD987 & 0.0 & 0.0 & double* \\
\hline 0006 & 001504.18 & -725028.9 & 1 & 1 & 0 & 0 & 0 & 0 & $\mathrm{M} 2 \mathrm{~V}$ & EXO 001239-7307.3 & 0.0 & 0.0 & flare* \\
\hline 0007 & 001821.34 & +305720.6 & 1 & 1 & 0 & 0 & 0 & 0 & G5 & HD1405 & 9.6 & 8.6 & RSCVn \\
\hline 0008 & 001915.27 & +074820.7 & 1 & 1 & 0 & 0 & 0 & 0 & $\mathrm{MU}$ & 1RXS J001915.4+074816 & 0.0 & 0.0 & \\
\hline 0009 & 002010.69 & +013304.7 & 1 & 1 & 0 & 0 & 0 & 0 & star & RBS44 & 0.0 & 0.0 & \\
\hline 0010 & 002123.26 & +334232.3 & 0 & 0 & 1 & 1 & 0 & 0 & starU & 1RXS J002123.2+334236 & 0.0 & 0.0 & \\
\hline 0011 & 002137.46 & -460527.6 & 1 & 1 & 0 & 0 & 0 & 0 & M3 & GJ3029 & 0.0 & 12.29 & $\mathrm{Fl}^{*}$ \\
\hline 0012 & 002354.30 & +605741.3 & 1 & 1 & 0 & 0 & 0 & 0 & $\mathrm{x}$ & 1RXS J002354.0+605743 & 0.0 & 0.0 & \\
\hline 0013 & 002405.22 & -720457.9 & 0 & 0 & 0 & 0 & 1 & 1 & & NuID & 0.0 & 0.0 & \\
\hline 0014 & 002502.18 & -452939.2 & 1 & 1 & 0 & 0 & 0 & 0 & Sy1 & ESO242-8 & 0.0 & 16.1 & \\
\hline 0015 & 002508.07 & +640955.7 & 1 & 1 & 0 & 0 & 0 & 0 & B8 & SNR021.0+63.0 & 0.0 & 0.0 & SNR \\
\hline 0016 & 002548.90 & +450132.6 & 1 & 1 & 0 & 0 & 0 & 0 & $\mathrm{X}$ & 1RXS J002548.8+450136 & 0.0 & 0.0 & \\
\hline 0017 & 002932.58 & -374131.0 & 1 & 1 & 0 & 0 & 0 & 0 & $\mathrm{~K} \mathrm{U}$ & 1RXS J002932.6-374133 & 0.0 & 0.0 & \\
\hline 0018 & 003509.19 & -502010.4 & 1 & 1 & 0 & 0 & 0 & 0 & K1III & HD3237 & 10.82 & 9.8 & \\
\hline 0019 & 003528.90 & +603137.7 & 1 & 1 & 0 & 0 & 0 & 0 & starU & 1RXS J003528.6+603139 & 0.0 & 0.0 & \\
\hline 0020 & 003803.93 & +790329.0 & 1 & 1 & 0 & 0 & 0 & 0 & & NuID & 0.0 & 0.0 & \\
\hline 0021 & 004031.30 & +520903.8 & 1 & 1 & 0 & 0 & 0 & 0 & star & 1RXS J004031.1+520906 & 0.0 & 0.0 & \\
\hline 0022 & 004111.89 & +552354.2 & 1 & 1 & 0 & 0 & 0 & 0 & $\mathrm{~K} \mathrm{U}$ & 1RXS J004111.8+552357 & 0.0 & 0.0 & \\
\hline 0023 & 004718.06 & -224503.7 & 1 & 1 & 0 & 0 & 0 & 0 & $\mathrm{~K} 4 \mathrm{~V}$ & RBS110 & 12.33 & 11.27 & \\
\hline 0024 & 004753.02 & -324521.3 & 1 & 1 & 0 & 0 & 0 & 0 & $\mathrm{~K}$ & CD-33 302 & 11.43 & 10.51 & \\
\hline 0025 & 005029.16 & +112858.6 & 1 & 1 & 0 & 0 & 0 & 0 & star & 1RXS J005029.2+112902 & 0.0 & 0.0 & \\
\hline 0026 & 005054.24 & -710916.4 & 0 & 0 & 0 & 0 & 1 & 1 & F5V & HD5028 & 7.274 & 6.85 & \\
\hline 0027 & 005238.18 & -185947.1 & 1 & 1 & 0 & 0 & 0 & 0 & & NuID & 0.0 & 0.0 & \\
\hline 0028 & 005308.28 & -743904.0 & 1 & 1 & 1 & 1 & 0 & 1 & G3 & $\mathrm{V} * \mathrm{CF}$ Tuc & 8.31 & 7.6 & RSCVn* \\
\hline 0029 & 005331.39 & +854633.7 & 1 & 1 & 0 & 0 & 0 & 0 & star & G265-4 & 15.3 & 0.0 & rotvar* \\
\hline 0030 & 005604.86 & +415318.7 & 1 & 1 & 0 & 0 & 0 & 0 & $\begin{array}{l}\text { star } \\
\text { starU }\end{array}$ & 1RXS J005604.8+415319 & 0.0 & 0.0 & \\
\hline 0031 & 005642.19 & +604259.2 & 1 & 1 & 0 & 0 & 0 & 0 & BoIVe & HD5394 & 2.29 & 2.39 & $\mathrm{Be}^{*}$ \\
\hline 0032 & 005655.94 & -751350.1 & 1 & 1 & 0 & 0 & 0 & 1 & gal & GSC09142-00531 & 0.0 & 0.0 & \\
\hline 0033 & 005715.92 & -824527.4 & 1 & 1 & 0 & 0 & 0 & 0 & star & 1RXS J005718.6-824526 & 0.0 & 0.0 & \\
\hline 0034 & 005720.34 & -222252.2 & 1 & 1 & 0 & 0 & 0 & 0 & Sy1 & 1AXG J005720-2222 & 0.0 & 0.0 & \\
\hline 0035 & 005836.67 & +842223.6 & 1 & 1 & 0 & 0 & 0 & 0 & $\mathrm{~K} \mathrm{U}$ & 1RXS J005834.5+842221 & 0.0 & 0.0 & \\
\hline 0036 & 010228.27 & +410159.2 & 1 & 1 & 0 & 0 & 0 & 0 & star & LP194-16 & 0.0 & 14.59 & \\
\hline 0037 & 010705.28 & +190904.9 & 1 & 1 & 0 & 0 & 0 & 0 & star & GSC01199-00701 & 10.95 & 9.94 & \\
\hline 0038 & 011154.39 & -754215.5 & 1 & 1 & 0 & 0 & 0 & 0 & IR N & & 0.0 & 0.0 & \\
\hline 0039 & 011229.74 & -165952.2 & 1 & 1 & 0 & 0 & 0 & 0 & M4.5 & $\mathrm{V} * \mathrm{YZ}$ Cet & 12.8 & 11.6 & $\mathrm{Fl}^{*}$ \\
\hline 0040 & 011240.58 & -291040.5 & 1 & 1 & 0 & 0 & 0 & 0 & KOIII & HD7280 & 9.68 & 8.8 & double* \\
\hline 0041 & 011319.68 & +585523.2 & 1 & 1 & 0 & 0 & 0 & 0 & starU & 1RXS J011319.6+585523 & 0.0 & 0.0 & \\
\hline 0042 & 011324.51 & -633833.0 & 1 & 1 & 0 & 0 & 0 & 0 & $\mathrm{X}$ & 1RXS J011323.2-633831 & 0.0 & 0.0 & \\
\hline 0043 & 011706.55 & -732635.0 & 1 & 1 & 0 & 0 & 0 & 1 & B0 & 2A $0116-737$ & 13.12 & 13.3 & Pulsar \\
\hline 0044 & 011800.14 & +651731.5 & 1 & 1 & 0 & 0 & 0 & 0 & B0.5I & $\mathrm{V} * \mathrm{~V} 662 \mathrm{Cas}$ & 0.0 & 0.0 & HMXB \\
\hline 0045 & 011836.72 & -732529.3 & 0 & 0 & 0 & 0 & 1 & 1 & GOIV & CCDM J01187-7325AB & 10.84 & 10.19 & double* \\
\hline 0046 & 012210.99 & +220905.5 & 1 & 1 & 0 & 0 & 0 & 0 & star & G34-23 & 15.1 & 0.0 & $\mathrm{PM}^{*}$ \\
\hline 0047 & 012250.52 & +004245.3 & 1 & 1 & 0 & 0 & 0 & 0 & G0 & $\mathrm{V} * \mathrm{BI}$ Cet & 9.01 & 8.29 & RSCVn \\
\hline 0048 & 012757.77 & +185924.9 & 1 & 1 & 0 & 0 & 0 & 0 & F8 & BD+18 193 & 9.86 & 9.36 & \\
\hline 0049 & 013117.34 & -395645.3 & 1 & 1 & 0 & 0 & 0 & 0 & gal N & 1RXS J013117.4-395648 & 0.0 & 0.0 & \\
\hline 0050 & 013126.09 & +360238.7 & 1 & 1 & 0 & 0 & 0 & 0 & star & RX J0131.4+3602 & 0.0 & 0.0 & cataclvar \\
\hline 0051 & 013240.66 & -080409.3 & 0 & 0 & 1 & 1 & 0 & 0 & $\mathrm{~K} \mathrm{U}$ & PKS 0130-083 & 0.0 & 0.0 & \\
\hline 0052 & 013243.38 & -655437.3 & 1 & 1 & 0 & 0 & 0 & 0 & star & V*CV Hyi & 0.0 & 20.0 & cataclvar \\
\hline 0053 & 013253.58 & +583640.3 & 1 & 1 & 0 & 0 & 0 & 0 & & NuID & 0.0 & 0.0 & \\
\hline 0054 & 013426.80 & +642647.8 & 1 & 1 & 0 & 0 & 0 & 0 & starU & 1RXS J013426.8+642651 & 0.0 & 0.0 & \\
\hline 0055 & 013500.68 & -295434.3 & 1 & 1 & 0 & 0 & 0 & 0 & $\mathrm{~K} 3 \mathrm{~V}$ & $\mathrm{~V} * \mathrm{BB} \mathrm{Scl}$ & 8.05 & 7.14 & Variable \\
\hline 0056 & 013514.21 & -071259.0 & 1 & 1 & 0 & 0 & 0 & 0 & & NuID & 0.0 & 0.0 & \\
\hline 0057 & 013523.26 & -272810.7 & 1 & 1 & 0 & 0 & 0 & 0 & gal N & 1RXS J013523.1-272807 & 0.0 & 0.0 & \\
\hline 0058 & 013557.28 & +231548.1 & 1 & 1 & 0 & 0 & 0 & 0 & $\mathrm{~K}$ & RX J0135.9+2316 & 0.0 & 0.0 & \\
\hline
\end{tabular}




\section{References}

Allen, C. W. 1973, University of London (The Athlone Press) Belloni, T., Hasinger, G., \& Izzo, C. 1994, A\&A, 283, 1037

Budding, E. 1985, Information. Bull. Variable Stars, 2779, 1

Catalan, M. S., Sarna, M. J., Jomaron, C. M., \& Connon Smith, R. 1995, MNRAS, 275, 153

Coates, D. W., Halprin, L., Sartori, P. A., \& Thompson, K. 1983, MNRAS, 202, 427

Donley, J. L., Brandt, W. N., Eracleous, M., \& Boller, T. 2002, AJ, 124,1308

Dupuis, J., Chayer, P., Vennes, S., Christian, D. J., \& Kruk, J. W. 2000, ApJ, 537, 977

Green, P. J., Ali, B., \& Napiwotzki, R. 2000, ApJ, 540, 992

Greiner, J., Voges, W., Boller, T., \& Hartmann, D. 1999, A\&A, 138, 441

Grupe, D., Thomas, H.-C., \& Beuermann, K. 2001, A\&A, 367, 470

Haisch, B., \& Schmitt, J. H. M. M. 1994, ApJ, 426, 716

Heidt, J., \& Wagner, S. J. 1998, A\&A, 329, 853
Jahreiss, H., Wielen, R., \& Fuchs, B. 1998, Acta Histor. Astron., 3, 171

Krautter, J., Zickgraf, F.-J., Appenzeller, I., et al. 1999, A\&A, 350, 743

Kürster, M., \& Schmitt, J. H. M. M. 1996, A\&A, 311, 211

Mittaz, J. P. D., Rosen, S. R., Mason, K. O., \& Howell, S. B. 1992, MNRAS, 258, 277

Ochsenbein, F., Bauer, P., \& Marcout, J. 2000, A\&A, 143, 23

Press, W. H., Teukolsky, S. A., Vetterling, W. T., \& Flannery, B. P. 1992 (Cambridge University Press)

Ritter, H., \& Kolb, U. 1998, A\&AS, 129, 83

Schmitt, J. H. M. M. 1994, ApJS, 90, 735

Stelzer, B., \& Neuhäuser, R. 2000, A\&A, 361, 581

Wichmann, R., Schmitt, J. H. M. M., \& Hubrig, S. 2003, A\&A, 400, 293

Zimmermann, H. U., Becker, W., Belloni, T., et al. 1994, Max-PlanckInstitut für extraterrestrische Physik, 85740 Garching, Germany, EXSAS user's guide, MPE report 257 